\title{
Bulletin Board
}
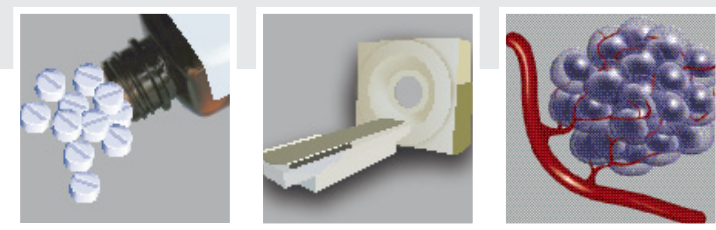

\section{Landmark ipilimumab approval is good news for inoperable or metastatic melanoma patients}

Promising overall survival data on ipilimumab (Yrevoy ${ }^{\mathrm{TM}}$; Bristol Meyers Squibb, NJ, USA) has paved the way for it to become the first unresectable or metastatic melanoma drug to be approved by the US FDA for over a decade. Ipilimumab manufacturer, Bristol Myers Squibb, announced the approval shortly after the publication of their pivotal data in the New England Journal of Medicine in March.

"Ipilimumab is the first in a new class of drugs that has been shown to offer a survival benefit for metastatic melanoma, which is often a fatal disease, and hopefully, this will lead to the development of related treatments for other cancers," said F Stephen Hodi, of the Dana-Farber Cancer Institute (MA, USA) and a lead investigator of the US clinical study of ipilimumab.

"For the first time, oncologists have a treatment option for patients with unresectable or metastatic melanoma that has been proven in a randomized Phase III clinical trial to significantly extend the lives of patients," said Steven J O'Day, from The Angeles Clinic and Research Institute (CA, USA), an investigator of the pivotal trial. "In fact, the Kaplan-Meier curve from this study suggests a prolonged survival benefit for some patients."

The FDA based their decision primarily on a randomized, double-blind study in 676 patients with unresectable or metastatic melanoma who were previously treated with one or more of a selection of chemotherapeutic agents. The patients were randomly assigned, in a 3:1:1 ratio, to receive ipilimumab plus gp100 (403 patients), ipilimumab alone (137), or gp100 alone (136). The Kaplan-Meier estimated survival rate at 1 year was $46 \%$ (95\% CI: $37.0,54.1)$ in the ipilimumab arm versus $25 \%$ (95\% CI: 18.1, $32.9)$ in the gp100 peptide vaccine arm. The estimated survival rate at 2 years was $24 \%$ (95\% CI: $16.0,31.5)$ in the ipilimumab arm versus $14 \%$ (95\% CI: 8.0, 20.0) in the gp100 arm. Median overall survival was 10
(95\% CI: 8.0-13.8), 10 (95\% CI: 8.5-11.5) and 6 (95\% CI: 5.5-8.7) months for the ipilimumab alone, ipilimumab plus gp100 arm and gp100 alone arms, respectively.

The approval came alongside a boxed warning for immune-related adverse reactions, which occurred in both arms treated with ipilimumab. Despite this, the drug will be welcomed by the community. Postmarketing data is anticipated on the safety and efficacy of a higher dosage.

Sources: Bristol Myers Squibb press release: www.bms.com/news/press_releases/pages/default. aspx; Hodi FS, O'Day SJ, McDermott DF et al.: Improved survival with ipilimumab in patients with metastatic melanoma. N. Engl. J. Med. 363, 711-723 (2010).

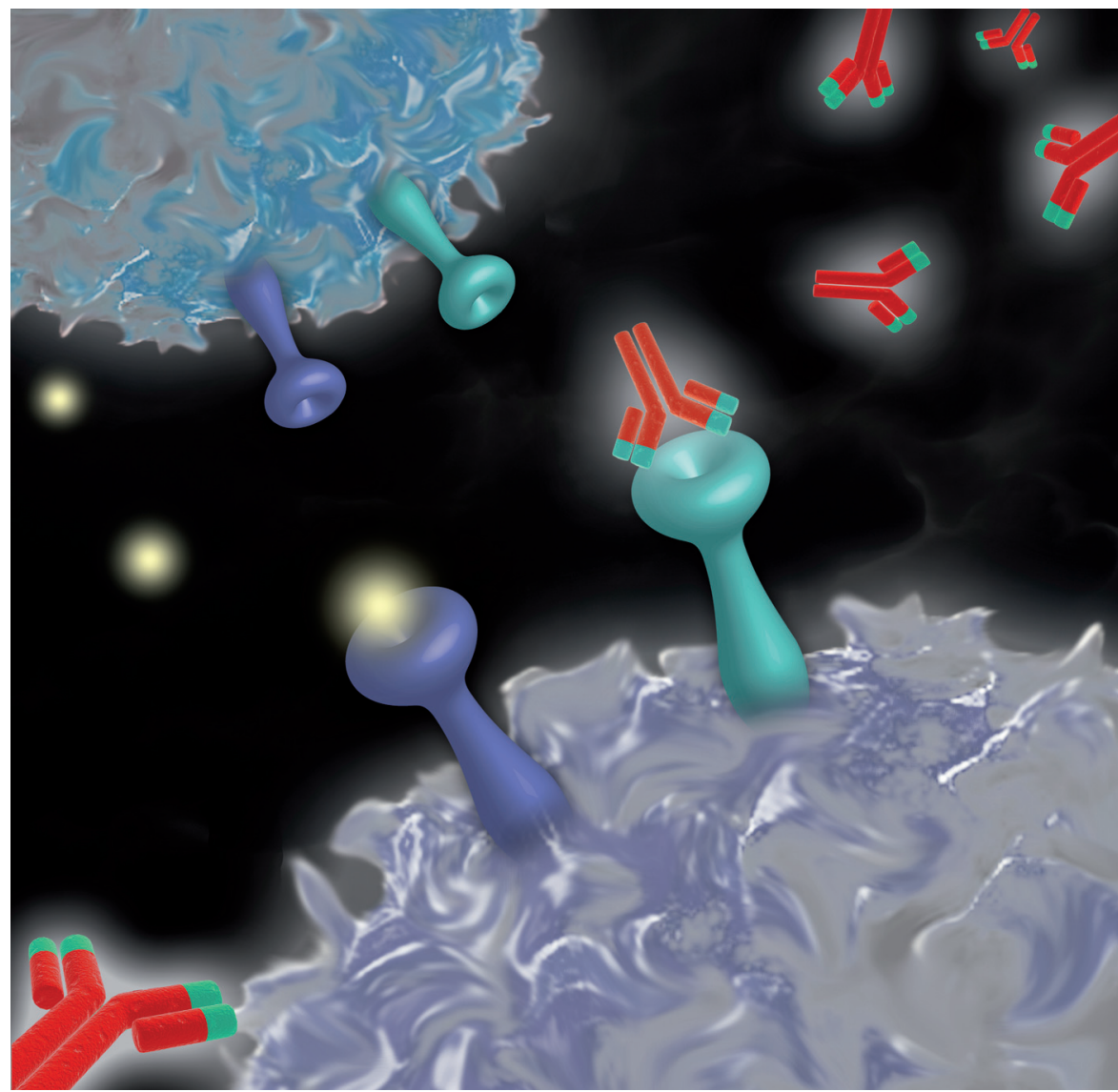




\section{Results of 5-year trial confirm that tamoxifen boosts breast cancer survival}

A 5-year trial, funded by Cancer Research UK, has demonstrated that cancer was less likely to return in women who took the drug for 5 years, when compared with those who took it for 2 years.

"This recent study ... found that cancer returned in $46 \%$ of the women who took tamoxifen for 2 years, and that it returned in $40 \%$ of women who took it for 5 years."

This recent study published in the Journal of Clinical Onocology, involving 3500 patients, found that cancer returned in $46 \%$ of the women who took tamoxifen for 2 years, and that it returned in $40 \%$ of women who took it for 5 years.

It had already been know that taking tamoxifen for 5 years increases the probability of survival from breast cancer. However, this recent study carried out by researchers from Cancer Research UK and University College London (UK) is the first large-scale study to compare the long-term benefit of tamoxifen for 5 years versus 2 years over a 10 -year follow-up period.

Lead author of this study, Allan Hackshaw (Univeristy College London), commented: "Our study provides conclusive evidence that taking tamoxifen for 5 years offers women the best chance of surviving breast cancer. Women diagnosed with early-stage breast cancer who are prescribed tamoxifen are recommended to take the drug for 5 years, but we know that many stop after 2 or 3 years. Worryingly our results suggest that by doing this, they could increase their risk of cancer coming back."

Another benefit found from taking tamoxifen for 5 years was that it reduced the risk of developing or dying from heart diseases. This effect was most pronounced for women aged 50-59 years at diagnosis, in this 5 -year group $35 \%$ fewer women developed a heart condition and approximately of $60 \%$ deaths occurred as a result of this.

Kate Law, director of clinical research at Cancer Research UK, said: "This large Cancer Research UK-funded study is the first to look at the long-term performance of tamoxifen - considered to be one of the most important drugs in the history of breast cancer treatment...It's vital that doctors and nurses continue encouraging women to finish their course of tamoxifen and providing the necessary support to ensure any side effects are effectively managed."

Sources: Hackshaw A, Roughton M, Forsyth S et al.: Long-term benefits of 5 years of tamoxifen: 10-year follow-up of a large randomized trial in women at least 50 years of age with early breast cancer. J. Clin. Oncol. DOI: 10.1200/JCO.2010.32.2933 (2011) (Epub ahead of print); Trial results confirm five years of tamoxifen boosts breast cancer survival: http:// info.cancerresearchuk.org/news/archive/ pressrelease/2011-03-21-five-years-of-tamoxifen

\section{Priority Paper Alerts}

Bravo-Cordero JJ, Oser M, Chen X, Eddy R, Hodgson L, Condeelis J: A novel spatiotemporal RhoC activation pathway locally regulates cofilin activity at invadopodia. Curr. Biol. DOI: 10.1016/j.cub.2011.03.039 (2011) (Epub ahead of print).

Rho GTPases have previously been associated with cancer metastasis through their alteration of the phosphorylation status of cofilin at invadopodia. Cofilin can regulate actin, producing actin-free barbed ends, and it is this element of the invadopodia that is crucial to drive invasion. This study conveys how it is the activation of RhoC at the invadopodia that enhances coflin phosphorylation and thus generates the actin-free barbed ends associated with cancer metastasis. The authors found that pl90RhoGEF localizes around the invadopodia to promote the activation of RhoC, whilst pl90RhoGAP localizes within the invadopodia, deactivating GTPase. Live cell imaging of fluorescent RhoC biosensor conveyed this restriction of RhoC around the invadopodia, which results in cofilin activity within the core of the invadopodia structure, consequently producing a focused invadopodia protrusion that is thought to promote tumor-cell invasion during metastasis.

Byrne KT, Côté AL, Zhang P et al.: Autoimmune melanocyte destruction is required for robust CD8+ memory T cell responses to mouse melanoma. J. Clin. Invest. DOI: 10.1172/JCl44849 (2011) (Epub ahead of print).

Melanoma-associated vitiligo occurs when immune cells attacking skin cancer also attack healthy menalocytes, resulting in cell destruc-
tion and patches of abnormal pigmentation. In the present study, the authors discovered that vitiligo generates a self antigen that is
required to mount a persistent and protective memory CD8+ T-cell response to melanoma. In a mouse model of melanoma, the group
found that $60 \%$ of immunonsuppressed mice that had undergone surgical removal of melanoma developed vitiligo. These mice were
found to generate ten times more CD8+ memory T cells specific for melanoma/melanocyte antigens. These memory cells provided
long-lasting tumor protection and were capable of mounting a rapid response to melanoma. Interestingly, the same responses were
not observed in melanocyte deficient mice, indicating a requirement for melanocyte destruction in maintaining CD8+ T-cell immunity
to melanoma. The authors conclude that their work demonstrates that immune-mediated destruction of normal tissues can lead to
an adaptive immune response to cancer. 


\section{Novel therapy stimulates the immune system to attack scaffolding surrounding pancreatic tumors}

Researchers have discovered a novel way to treat pancreatic cancer, utilizing the patient's own immune system to destroy the scaffolding surrounding the tumor.

The results were observed in an ongoing study that utilizes a unique research model designed to improve understanding of effective treatment options. The trial involved pancreatic cancer patients receiving standard gemcitabine chemotherapy with an experimental antibody that binds and stimulates the cell surface receptor CD40, a key regulator of T-cell activation.

The team hypothesized that the experimental antibodies would turn on the $\mathrm{T}$ cells, allowing them to attack the tumor, and the treatment appeared effective, with some patients' tumors shrinking substantially, as well as the vast majority losing metabolic activity after treatment. However, all of the patients who responded to initial treatment did eventually relapse. On inspection of the tumor samples after treatment, the researchers noted the absence of $\mathrm{T}$ cells and an abundance of macrophages.
This unusual observation prompted them to revert back to an earlier mouse model of pancreatic cancer developed several years previously. Treating mice with the same gemcitabine and experimental antibody therapy resulted in similar effects in reducing tumor size and allowed the researchers to further investigate the macrophage abundance. The macrophages appeared to be attacking the tumor stroma, which surrounds the tumor to provide support. Pancreatic tumors secrete chemical signals to draw macrophages to the site, which would normally provide protection to the tumor. However, treatment with the CD40 antibodies appears to have the opposite effect. "It is something of a Trojan horse approach," commented Robert Vonderheide, associate professor of medicine at the Abramson Family Cancer Research Institute (PA, USA). "The tumor is still calling in macrophages, but now we've used the CD40 receptor to re-educate those macrophages to attack - not promote - the tumor."

The researchers believe that the CD40 antibodies also activated $\mathrm{T}$ cells in the mice, but they were unable to reach the tumor or its surrounding tissue owing to the dense, fibrotic area surrounding pancreatic cancers. "We learned that $\mathrm{T}$ cells have a major problem with migration into tumors, and this may be a particular problem for pancreatic cancer. The area surrounding pancreatic cancers is very dense, fibrotic, and hostile. This is one of the main reasons standard therapies for this disease often work so poorly," continued Vonderheide.

The researchers are testing ways to maximize the macrophage response and enabling the $T$ cells to reach the tumor microenvironment. "Attacking the dense tissues surrounding the cancer is another approach, similar to attacking a brick wall by dissolving the mortar in the wall. Ultimately, the immune system was able to eat away at this tissue surrounding the cancer, and the tumors fell apart as a result of that assault. These results provide fresh insight to build new immune therapies for cancer," said Vonderheide.

Source: Penn Medicine News: www.uphs. upenn.edu/news/News_Releases/2011/03/ pancreatic-cancer-immunotherapy

\section{Targeted antiglycolytic treatment for breast cancer suggested}

New research presented at the Society of Interventional Radiology's 36th Annual Scientific Meeting in Chicago, IL, USA, suggests that treatment combining a strategy that blocks an enzyme in the cancers glycolysis pathway with infusion of a drug directly into the tumor may help to delay the growth and spread of breast cancer.

Jeff Geschwind (Johns Hopkins University School of Medicine, Baltimore, MD, USA), lead researcher, noted of the study, "Once breast cancer metastases have been detected, current treatments (such as surgical resection or tumor removal) may be ineffective. We've found a way to keep a breast cancer tumor dormant - thus potentially increasing the likelihood that a tumor can be treated successfully..."
Breast tumor cells depend on a metabolic pathway called glycolysis to generate the energy required for their malignant growth. By inhibiting a specific enzyme with the antiglycolytic agent 3-bromopyruvate $(3-\mathrm{BrPA})$, the energy production required for tumor cell growth and spread is blocked," The group were able to show that 3-BrPA effectively disrupted glycolysis so that cancer cells were unable to produce energy and survive.

The group used animal studies to show that minimally invasive insertion of 3-BrPA using an ultrasound guided intratumoral approach can effectively delay the growth and spread of tumors. As Geschwind notes, "The biological targeting abilities of antiglycolytic treatment combined with an image-guided minimally invasive delivery strategy is a promising approach to reducing the growth and spread of breast cancer in patients."

The combination of these two novel strategies could, if verified in human trials, significantly improve treatment options for breast cancer patients

"In our study, a statistically significant difference in tumor volume was observed. Our results support the continuing development of this highly innovative interventional radiology approach for the safe and effective treatment of breast cancer," concluded Geschwind.

\footnotetext{
Source: Liapi E, Geschwind JF: Medium-sized HCC: achieving effective local tumor control with combined chemoebolization and radiofrequency ablation. Ann. Surg. Oncol. DOI: 10.1245/s10434-011-1679-2 (2011) (Epub ahead of print).
} 